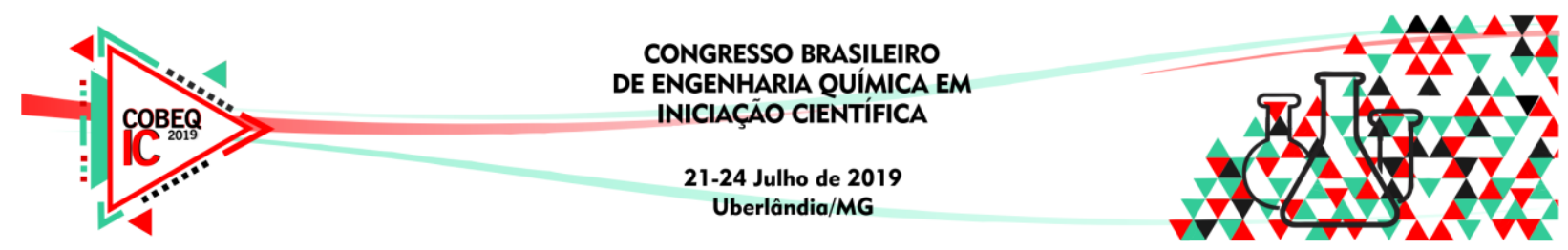

\title{
CARACTERIZAÇÃO DE SOLUÇÕES POLIMÉRICAS INCORPORADAS COM ÁCIDO SALICÍLICO PARA OBTENÇÃO DE NANOFIBRAS ELETROFIADAS
}

\author{
R. L. M. SOUZA ${ }^{1}$, B. A. ROCHA ${ }^{1}$ e J. V. W. SILVEIRA ${ }^{1}$ \\ - Universidade Federal dos Vales Jequitinhonha e Mucuri, Instituto de Ciência e Tecnologia \\ Diamantina-MG, Brasil. \\ E-mail para contato: rluiz.rluiz@ hotmail.com
}

\begin{abstract}
RESUMO - A ampla aplicabilidade de materiais nanopoliméricos fez surgir inúmeros estudos com o intuito de analisar e otimizar suas propriedades e funções. Realizou-se neste estudo a caracterização de soluções poliméricas com ácido salicílico (AS) e a capacidade das mesmas na formação de nanofibras eletrofiadas. A partir de uma solução padrão composta por $15 \%$ de acetato de celulose e $85 \%$ de uma mistura contendo $90 \%$ de acetona e $10 \%$ de dimetil sulfóxido, foram incorporadas quantidades distintas de AS, entre 10 e 50\% em relação à quantidade de polímero. Para caracterização das soluções utilizaram-se os testes de viscosidade e condutividade elétrica. Em seguida as soluções foram eletrofiadas e as fibras produzidas, submetidas à técnica de microscopia eletrônica de varredura. Os resultados obtidos nos testes de viscosidade e condutividade elétrica, apresentaram comportamento como o esperado. Já no caso da eletrofiação, as soluções contendo $10 \%$ e $20 \%$ apresentaram as melhores formações de fibras. Uma hipótese para que isso possa ter acontecido é pelo fato da presença, em grandes quantidades, de um material não polimérico, no caso o AS, desfavorecer a formação das nanofibras. Estudos posteriores serão realizados com o intuito de comprovar tais hipóteses.
\end{abstract}

\section{INTRODUÇÃO}

A produção de materiais poliméricos em tamanhos nanométricos está sendo atenciosamente estudados e trabalhados em vista das inúmeras propriedades associadas às possíveis novas aplicações. Dentre os materiais mais estudados destacam-se as nanofibras ou nanofios considerados promissores em inúmeras áreas para aplicação (COSTA et al., 2011).

Dentre outros métodos de produção das nanofibras, o método de eletrofiação se destaca efetivamente, isso ocorre devido a simplicidade e versatilidade do processo quando voltado a obtenção e fibras de diâmetro micro e nanométricos. Esse processo possui um arranjo basicamente formado por uma fonte de alta tensão, solução polimérica e um sistema de aterramento (COSTA et al., 2011; DOGNANI, 2016). O processo de formação das nanofibras consiste na ejeção da solução polimérica carregada em uma seringa por meio de uma bomba infusora. O êmbulo ao ser pressionado proporciona a formação de uma gota na ponta da 


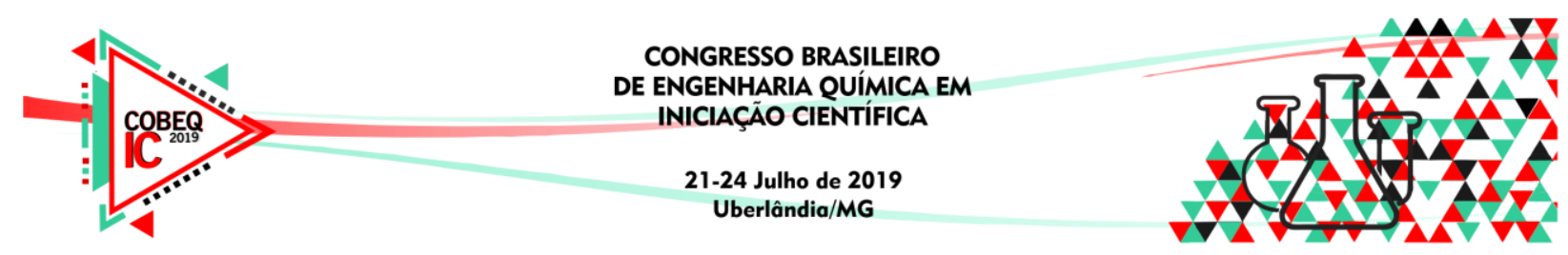

agulha. A diferença de potencial existente entre a extremidade da agulha e do coletor metálico faz com que a gota formada se alongue obtendo um formato de cone, conhecido como cone de Taylor. Ao aumentar a tensão elétrica a superfície da gota é conduzida ao coletor e moldada em fios extremamente finos (CARMO; MEDEIROS; MEDEIROS, 2000; AMMAR; ZAGROUBA; ROMDHANE, 2010; DOGNANI, 2016).

As soluções poliméricas dependem do tipo de aplicabilidade que terá as fibras a serem produzidas. No caso do presente trabalho, serão utilizadas para aplicação dermatológica, com isso cada componente da solução tem importante função para maior eficácia das fibras. $\mathrm{O}$ acetato de celulose foi usado como biopolímero devido ao polímero neutro que ele forma, sua característica de ser matriz para liberação de fármacos e possuir um baixo custo (CERQUEIRA et al., 2009). Os solventes utilizados nos processos de eletrofiação tem papel importante nas características morfológicas das fibras, quando são muito voláteis acabam evaporando rapidamente o que origina um aumento considerável na viscosidade e consequentemente no diâmetro dos fios. Ainda podem influenciar na solubilidade do polímero, tensões superficiais e até mesmo na condutividade elétrica. Dessa forma, foram escolhidos a acetona e dimetilsulfóxido (DMSO) por apresentarem boa eficiência no processo de interesse. O DMSO ainda apresenta ação anti-inflamatório o que o deixa ainda mais interessante para a aplicação de interesse (RAMOS, 2011; SOUZA; ORÉFICE, 2016). O fármaco agregado a solução foi o ácido salicílico (AS), devido seu excelente resultado no combate a acne. $\mathrm{O}$ ácido salicílico é um dos mais populares fármacos para esta aplicação devido suas propriedades antimicrobiana e anti-inflamatória, que reduz a produção sebácea, população de bactérias e processos inflamatórios (LIMA; DELAY, 2017). Neste trabalho estudou-se a formação das fibras a partir de soluções com diferentes concentrações de AS, com o objetivo de avaliar a formação das fibras.

\section{METODOLOGIA}

Para a produção das fibras foram utilizados acetato de celulose $(\mathrm{Mw} \sim 30.000$, SigmaAldrich, Brasil); ácido salicílico P. A. (Synth, Brasil); acetona (Quimis, Brasil) e dimetilsulfóxido (Quimis, Brasil). O aparato de eletrofiação conta com uma bomba seringa (Harvard Apparatus, EUA) e fonte de alta tensão (Faíscas, Brasil).

\subsection{Produção das soluções poliméricas}

O procedimento inicial consistiu na produção das soluções poliméricas a serem fiadas para a formação das nanofibras. A solução inicial, denominada solução padrão, é composta por $15 \%$ de acetato de celulose e $85 \%$ de uma mistura contendo $90 \%$ de acetona e $10 \%$ de DMSO, os quais foram adicionados em um recipiente e mantidos em agitação no agitador magnético por 24 horas. A partir de tal solução padrão, preparou-se outras cinco soluções adicionado ácido salicílico em porcentagens distintas em relação ao polímero já existente na solução padrão. Tais porcentagens foram distribuídas de forma que a solução 1 contém 10\% de ácido salicílico e $90 \%$ de acetato de celulose, a solução 2 possui $20 \%$ de ácido salicílico e $80 \%$ de acetato de celulose, e assim por diante, até que a quinta solução produzida contém $50 \%$ de ácido salicílico e $50 \%$ de acetato de celulose. Cada uma dessas soluções está armazenada em vidrarias devidamente seladas e etiquetadas. 


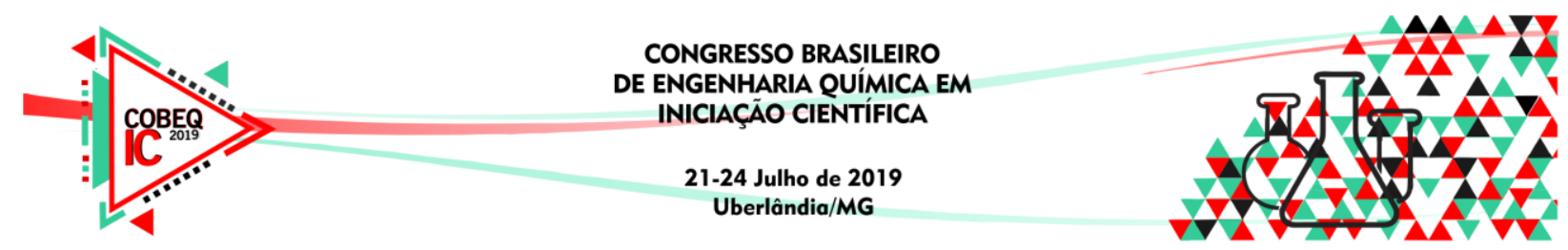

\subsection{Teste de viscosidade}

Os testes de viscosidade foram realizados com as soluções em temperatura ambiente, por volta de $25{ }^{\circ} \mathrm{C}$. Utilizando o viscosímetro Brookfield DV-III Ultra, cada uma das seis amostras foi colocada em provetas de $10 \mathrm{~mL}$ e utilizando o spindle 64 do modelo LV. As faixas de rotação consideradas foram aquelas onde os valores de viscosidade estiveram mais estáveis a partir do spindle selecionado.

\subsection{Produção das nanofibras}

Para a produção das nanofibras utilizou-se a técnica eletrofiação. Onde o arranjo experimental utilizado é composto por uma fonte de alta tensão com $15 \mathrm{kV}$, um aparato de metal com $15 \mathrm{~cm}$ de diâmetro, coberto de papel laminado para o depósito das fibras, uma agulha de $10 \mathrm{~mL}$ e uma bomba infusora ligada a um controle de liberação realizado pelo programa WinPump; aparato demonstrado na Figura 1.

Figura 1 - Arranjo experimental utilizado em eletrofiação

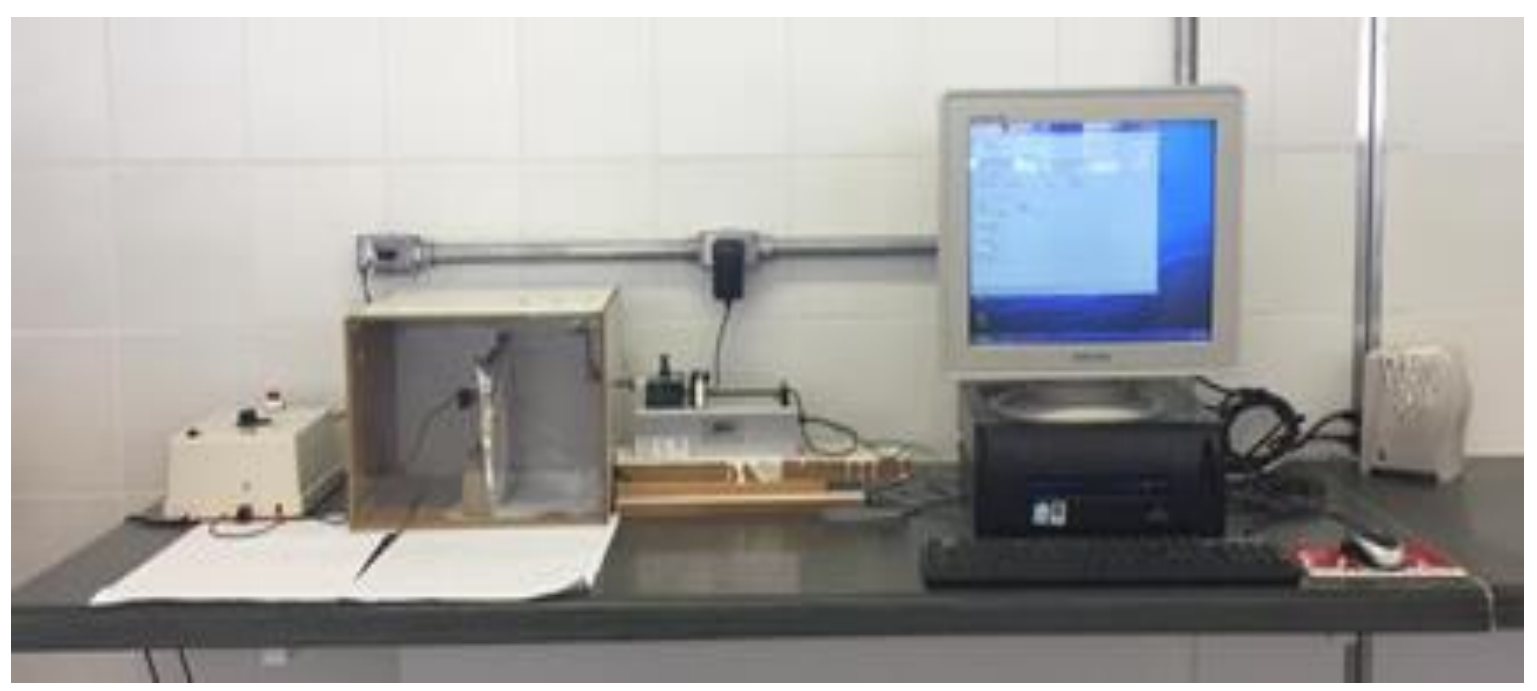

Cada uma das soluções previamente produzidas foi fiada por aproximadamente 30 minutos, com o intuito de viabilizar a formação de nanofibras com as distintas quantidades de ácido salicílico, 0\%, 10\% 20\% 30\% 40\% e 50\%.

\subsection{Microscopia eletrônica de varredura}

Os testes de microscopia eletrônica de varredura (MEV) foram realizados nas amostras fiadas, com o intuito de comprovar a formação ou não formação das fibras nas mesmas. Para a realização deste teste, cortou-se aproximadamente um quadrado com $0,5 \mathrm{~cm}^{2}$ de cada amostra fiada e posicionou-se corretamente no aparelho, HITACHI TM3000 Tabletop Scanning Electron Microscope. Ao final do procedimento, para cada uma das amostras analisadas, obteve-se imagens microscópicas com ampliações de 500 a 10.000 x. 


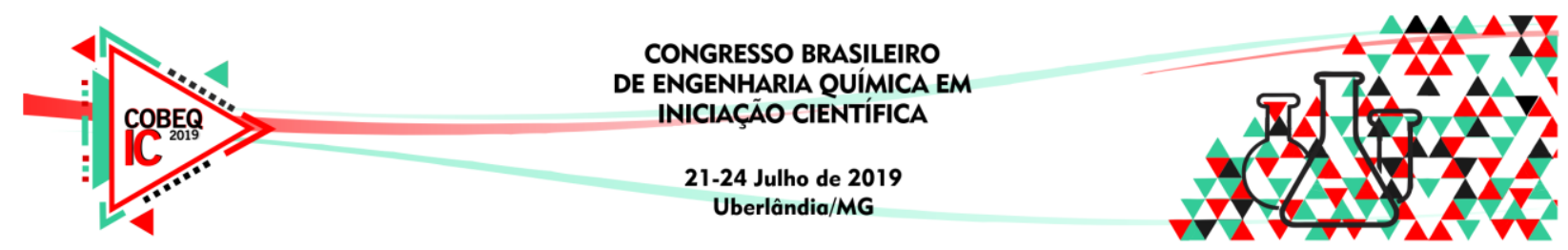

\section{RESULTADOS}

A partir das soluções produzidas, os resultados obtidos nos testes de condutividade elétrica e de viscosidade foram expostos em forma de gráfico, conforme a Figura 2.

Figura 2 - Análises de condutividade elétrica e viscosidade realizadas nas soluções.

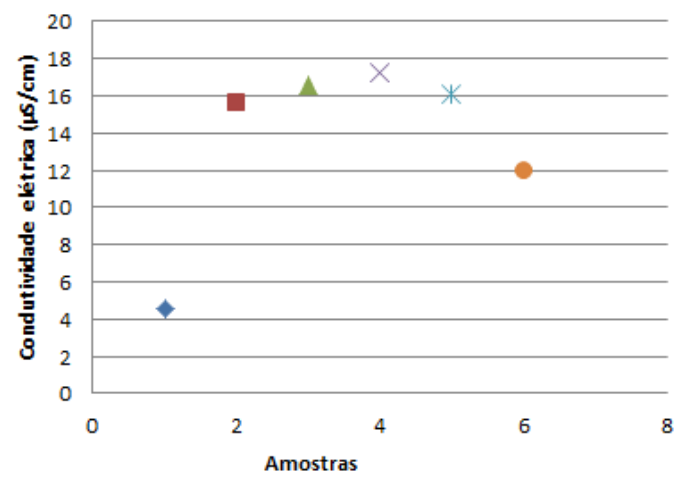

(a) Teste de condutividade elétrica

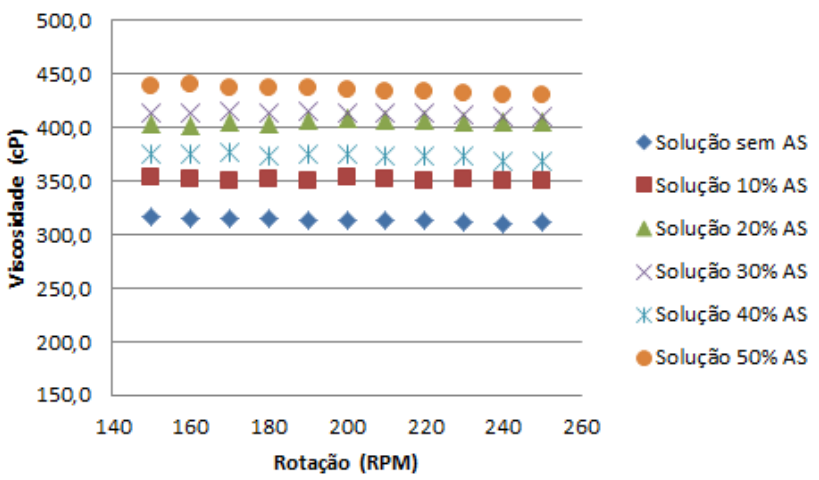

(b) Teste de viscosidade

De acordo com Ramakrishna e Fujihara (2018) é esperado que ao aumentar a quantidade de sólidos na solução, sua massa crescerá e, com isso, a viscosidade também. Os resultados do teste de viscosidade seguem essa lógica, como demonstrado na Figura 2 (B), porém a solução de $40 \%$ mostrou um comportamento inesperado. Tal resultado pode estar relacionado a pequenas variações de temperatura que podem ter ocorrido no decorrer dos testes. Já o teste de condutividade, apresentou um comportamento incomum. Ainda de acordo com Ramakrishna e Fujihara (2018) é esperado que ao aumentar a quantidade de íons livres com a adição de ácido, a condutividade da solução se eleve, como ocorreu para as soluções com 10, 20 e 30\% de AS. Porém, como é possível verificar na Figura 2 (a), no ponto 4 houve uma mudança de comportamento. As soluções de 40 e $50 \%$ apresentaram menor valor de condutividade. Uma das hipóteses levantadas que será investigada é que possivelmente a quantidade de cargas elétricas se tornou muito grande e com isso tornou instável a solução, além de possíveis interações que podem ter ocorrido entre o ácido e solvente ou polímero.

Após o procedimento de eletrofiação das soluções, realizou-se o teste de MEV em cada amostra. As Figuras 3 a 8 apresentam o resultado do MEV (para uma ampliação de 5000 x) para a solução padrão, solução com 10\%, 20\%, 30\%, 40\% e 50\% de AS, respectivamente. Conforme os resultados obtidos no MEV podemos observar que as soluções padrão, $10 \%, 20 \%$ apresentaram as melhores formações de nanofibras, ou seja, fibras bem consolidadas e com um baixo número beads (formação de bolhas poliméricas ao longo da fibra), sendo essas com diâmetros na faixa de $150 \mathrm{~nm}$ a $827 \mathrm{~nm}$. A solução de $30 \%$ apresenta a formação de fibras pouco definidas e beads bem estruturados, por isso não se considera essa fibra bem eletrofiação, ainda assim houve a formação da mesma. Para as soluções de $40 \%$ e $50 \%$ não se verifica a formação de fibras, isso ocorre devido ao fato do processo de eletrofiação, não ter ocorrido efetivamente. A presença de materiais não poliméricos em grande quantidade na solução, neste caso, o AS pode ter causado a desestruturação da solução, pois este material não eletrofiável desfavorece a formação da fibra. 


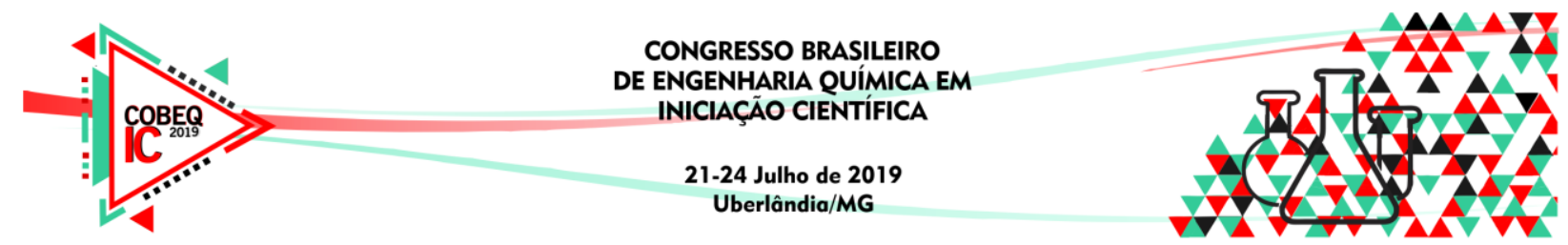

Figura 3 - MEV solução padrão sem AS

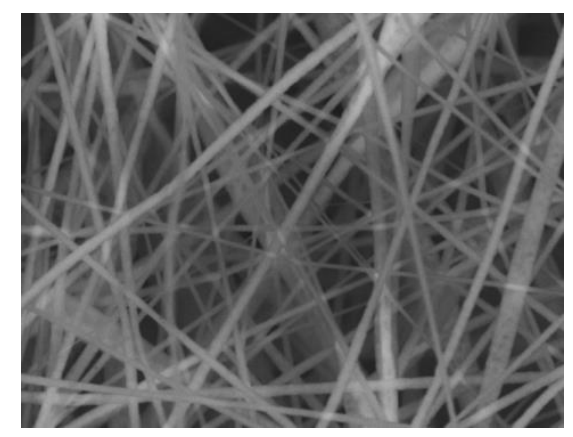

Figura 5 - MEV solução com $20 \%$ AS

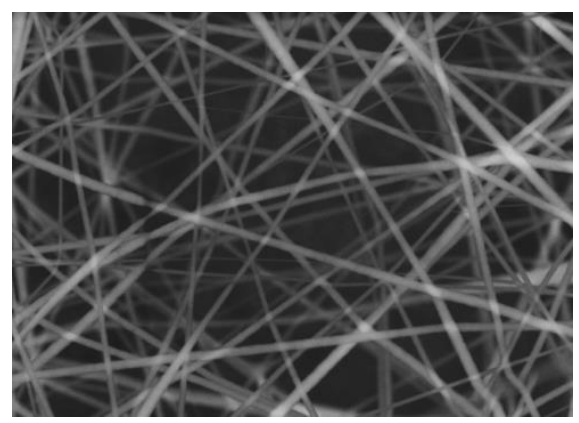

Figura 7 - MEV solução com $40 \%$ AS

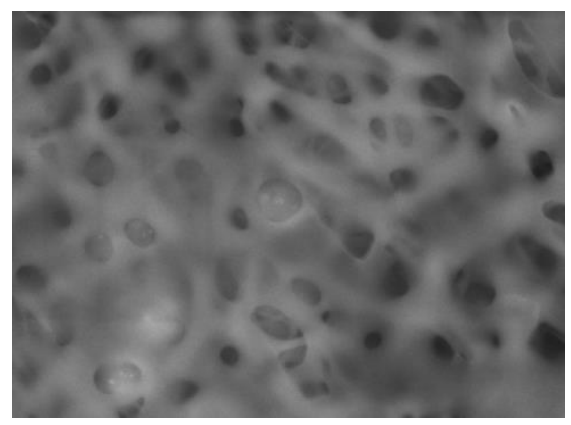

Figura 4 - MEV solução com $10 \%$ AS

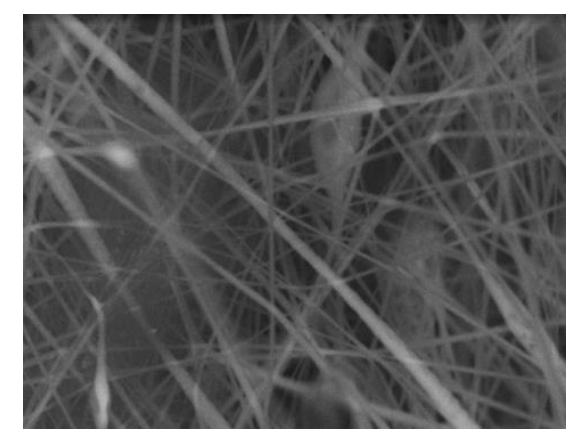

Figura 6 - MEV solução com $30 \%$ AS

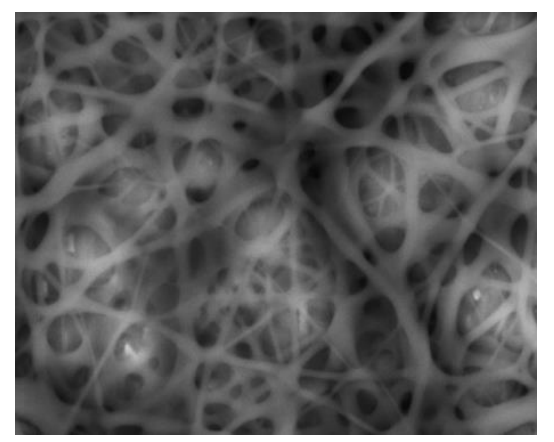

Figura 8 - MEV solução com 50\% AS

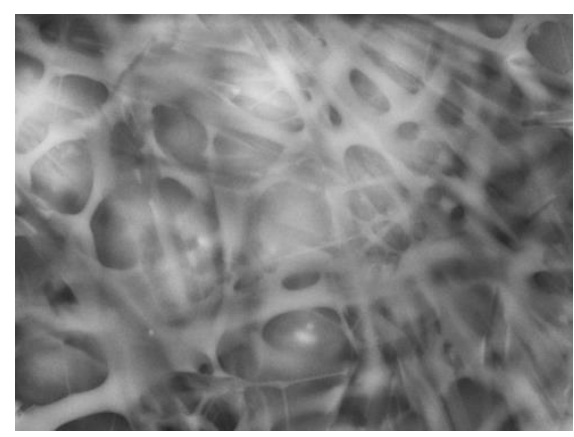

\section{CONCLUSÃO}

Pelos testes realizados ficou claro como as características da solução influencia diretamente na formação das fibras. Uma vez que ao adicionar mais um composto com características ácidas e com peso molecular considerável, obteve-se dificuldades na formação das nanofibras. Fica claro que a adição de um novo sólido modificou a viscosidade e condutividade da solução. Ainda é possível perceber que pelo fato do AS não ser um material que forma fibras, quando ele está presente em maior quantidade a formação dos fios não ocorreu. As hipóteses levantadas em torno da condutividade e da possível reação do AS com o solvente e/ou polímero ainda serão investigadas e analisadas futuramente. 


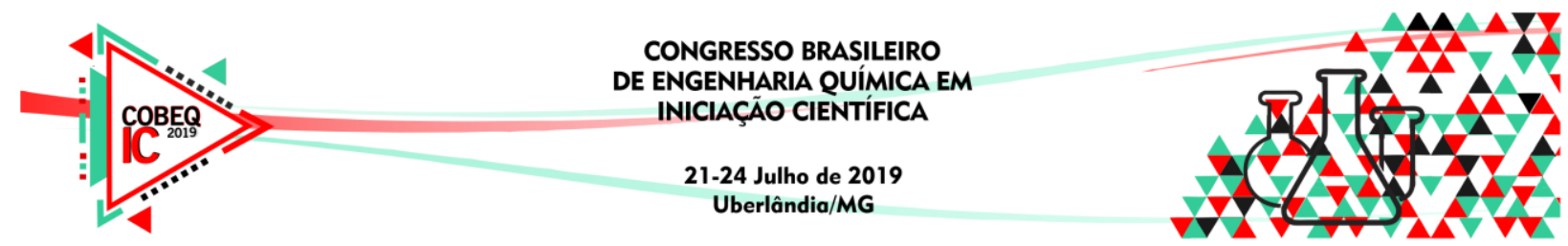

Os autores agradecem pelo suporte as agências CNPq e a UFVJM. E ainda, ao LMMA patrocinado pela FAPEMIG (CEX-112-10), SECTES/MG e RQ-MG (FAPEMIG: CEX-RED-00010-14).

\section{REFERÊNCIAS}

AMMAR, A. H.; ZAGROUBA, F.; ROMDHANE, M. Optimization of operating conditions of Tunisian myrtle (Myrtuscommunis L.) essential oil extraction by a hydrodistillation process using a 24 complete factorial design. Flavour and Fragrance Journal, v. 25, n. 6, p. 503-507, 2010.

CARMO, L. A.; MEDEIROS, A.; MEDEIROS, C. F. de. Distorções Conceituais em Imagens de Livros Textos: O Caso do Experimento de Joule com o Calorímetro de Pás. n. Vii, p. 13-14, 2000. Disponível em: <http://www.if.ufrgs.br/ lang/Textos/AlexMed/Exp_Joule.pdf>.

CERQUEIRA, D. A.; FILHO, G. R.; CARVAlHO, R. A.; VAlENTE, A. J. M. Caracterização de Acetato de Celulose Obtido a partir do Bagaço de Cana-de-Açúcar por 1 H-RMN. p. 7, 2009. Disponível em: <http://www.scielo.br/pdf/po/2010nahead/aop_0569.pdf>. Acesso em: 11 dez. 2018.

COSTA, R.G. F.; OLIVEIRA, J. E. PAULA, G. F.; PAULO, H.; PICCIANE, S.; CAUÊ, R.; MATTOSO, L. H. C. Eletrofiação de Polímeros em Solução. Parte I: Fundamentação Teórica. Polímeros: Ciência e Tecnologia, São Carlos, v.22, n. 8, p. 170-177, 2011.

LIMA, K. M. R.; DELAY, C. E. Utilização do ácido salicílico no tratamento da acne. [s.l: s.n.]. Disponível em: <https://tcconline.utp.br/media/tcc/2017/05/UTILIZACAO-DOACIDO-SALICILICO-NO-TRATAMENTO-DA-ACNE.pdf>. Acessoem: 13 abr. 2019.RAMAKRISHNA, S.; FUJIHARA, K. An Introduction to Electrospinning and Nanofibers. London: British Library Cataloguing-in-Publication Data, 2005.RAMOS, S. L. Membranas de Policaprolactona Obtidas por Eletrofiação para Utilização em Engenharia Tecidual. 2011.

SOUZA, S. O. L. de; ORÉFICE, R. L. Desenvolvimento e Caracterização de Nanofibras Obtidas pela Técnica de Eletrofiação Coaxial Visando a Liberação de Bevacizumabe para o Tratamento de Degeneração Macular Relacionada a Idade. 2016. 32016. Disponível em: $<$ https://www.bertelsmannstiftung.de/fileadmin/files/BSt/Publikationen/GrauePublikationen/MT_Globalization_R eport_2018.pdf\%0Ahttp://eprints.1se.ac.uk/43447/1/India_globalisation\%2C societyand inequalities\%281sero\%29.pdf\%0Ahttps://www.quora.com/What-is-the>. 